\title{
CONSTRUCTION OF EQUIVALENT MARTINGALE MEASURES WITH INFINITESIMALS
}

\author{
JIŘÍ WITZANY
}

\begin{abstract}
Equivalent martingale measures are of key importance for pricing of complex derivative contracts. The goal of the paper is to apply infinitesimals in the non-standard analysis set up to provide an elementary construction of the equivalent martingale measure built on hyperfinite binomial trees with infinitesimal time steps.
\end{abstract}

\section{INTRODUCTION}

A martingale is a stochastic process $M(t)$ such that $M(0)=E[M(t)]$ for every $t$. If $M(t)=\frac{f(t)}{g(t)}$ is a ratio of prices of a new derivative and of a positively valued derivative we already know how to price (numeraire) and if $M(t)$ is a martingale then $f(0)$ can be obtained as $g(0) \cdot E\left[\frac{f(T)}{g(T)}\right]$ as long as we are able to calculate analytically or numerically the expected value at certain future time $T$. The time $T$ is typically the expiration time of a European type derivative when the value simply equals to the payoff of the derivative. The most popular numeraire is the time $t$ market value $P(t, T)$ of the unit zero coupon bond maturing at time $T$. The main advantage is that if $M(t)=\frac{f(t)}{P(t, T)}$ is a numeraire then $f(0)=P(0, T) \cdot E[f(T)]$ since $P(T, T)=1$. The numeraire is in particular useful for valuation of interest rate derivatives where we simple must work with the fact that interest rates are stochastic (not constant as in the basic Black-Scholes model). Nevertheless it is clear that the fraction will rarely be a martingale in the real financial world where investors require higher return for higher risk. If we were able to adjust the measure under which the expected values are taken so that the process becomes a martingale then there is a chance that the valuation could be realized.

The equivalent martingale measure theorem provides exactly what we need. It says roughly speaking that if $g(t)$ is a numeraire, i.e. a stochastic process attaining positive values and modelling prices of a derivative security, then there is a measure $Q$ equivalent to the original measure $P$ such that $M(t)=\frac{f(t)}{g(t)}$ is a martingale with

Date: July, 2008.

2000 Mathematics Subject Classification. Primary 60H05, 60J65, 02H25.

Key words and phrases. Equivalent martingale measure, option pricing, stochastic processes, nonstandard analysis.

The author was supported in part by Czech Science Foundation grant \#402/06/0890. 
respect to $Q$. In the context of the pricing method explained above this is the key result for valuation of various derivative contracts like interest rate, quanto, in arrears etc. (see Hull (2006)).

The theorem is often applied and even heuristically proven without mentioning rigorous foundations of stochastic processes like $\sigma$-algebras, filtrations, etc. The standard mathematical theory behind the technique is indeed quite technically difficult. It should be noted that the famous Black-Scholes formula has been discovered using heuristic arguments without the modern rigorous theory of stochastic processes. The stochastic theory has been developed ex post partially in order to give a precise foundation to the Black-Scholes theory. We are going to built equivalent martingale measures in an elementary set up on hyperfinite binomial trees applying the methods of Cox, Ross, and Rubinstein (1979) but with infinitesimal time steps. We follow the paper of Cutland, Kopp, and Willinger (1991) but generalize the approach to a general numeraire with mutiple sources of uncertainty and also simplify the construction eliminating the notion of filtration building the processes step-by-step on binomial trees. We believe that the approach provides an intuitive framework on which further modelling concepts like processes with stochastic volatility could be easily built.

The set of real numbers can be extended with infinitesimals satisfying most of their intuitive properties using a technique discovered by A.Robinson (1961). The field of mathematics is called Non-Standard Analysis (NSA). It allows for example to differentiate and integrate using infinitesimals in an intuitive manner, but compared to the historical critism on rigorous foundations within Zermelo-Fraenkel Set Theory with the Axiom of Choice. The probability theory has been in particular developed by P.Loeb (1975) and foundation of stochastic processes with infinitesimals were given by Anderson (1976) and J.Keisler (1984). The elementary Brownian motion is in the standard approach constructed as a limit of approximating random processes. This approach makes undestanding of this basic stochastic process quite difficult. On the other hand with infinitesimals we can define the Brownian motion sequentially step by step "tossing a coin" to go up or down in line with our intuition. The result will be not an approximation but a perfect Browninan motion if infinitesimal time steps are used. This allows us to model similarly step by step financial processes like stock price development, trading, or hedging strategies in a way close to the financial markets reality.

The first application of this approach to derivetives pricing was given by Cutland, Kopp, and Willinger (1991). The paper gives a nonstandard proof of the classical Black-Scholes result and nonstandard treatment of self-financing hedging strategies. In subsequent papers Cutland, Kopp, and Willinger (1995) focus rather on the issue of $\left(D^{2}\right)$ convergence of finite Cox-Ross-Rubinstein approximations. The technique 
is applied to some particular derivative pricing problems like pricing of options with underlying assets following a Poisson jump process (1993) or to Snell envelopes for pricing of American put options (with Wyman (1997)).

We will extend the construction of a hyperfinite Cox-Ross-Rubinstein binary tree to the case of derivatives with multiple sources of uncertainty and with stochastic drift and volaitilties. We will use an intuitive technique building the processes step-by-step on hyperfinite trees that allows us to eliminate the notion of filtration.

\section{NONSTANDARD ANALYSIS - AN OVERVIEW}

For a detailed overview of NSA see for example Hurd and Loeb (1985) or the elementary textbook by Keisler (2000). Let us however review the basic principles.

There is (in the universe of sets with Axiom of Choice) an extension ${ }^{*} R$ of the real line $R$ with a number of properties outlined below. The extension ${ }^{*} R$ includes elements defined as non-zero "infinitesimals" $\left(x \in^{*} R\right.$ satisfying $|x|<\epsilon$ for all $\epsilon>0$ in $R$ ) and their "infinite" multiplicative inverses. The extension itself is not unique: ${ }^{*} R$ can, for example, be defined as an ultrapower $R^{N} / \mathcal{U}$ of the reals by any non-principal ultrafilter $\mathcal{U}$ on $N$ (i.e. a collection of subsets of $N$ closed under intersections and supersets, containing no finite sets, and such that for any $A \subseteq N$, either $A$ or $N-A$ belongs to $U$.) The existence of such ultrafilters is equivalent to the Axiom of Choice. What is important here is that the arithmetical and order operations valid in $R$ extend to ${ }^{*} R$ : the tuple $\left({ }^{*} R ;+; \times ;<\right)$ is an ordered field.

For any set $S$ the superstructure $V(S)$ over $S$ is defined as $V(S)=\cup_{n=0}^{\infty} V_{n}(S)$; where $V_{0}(S)=S$; and

$$
V_{n+1}(S)=V_{n}(S) \cup P\left(V_{n}(S)\right)(n \in N)
$$

This construction can be applied to $R$ as well as to ${ }^{*} R$. The superstructures $V=$ $V(R)$ and $V\left({ }^{*} R\right)$ are connected by a map $*: V(R) \rightarrow V\left({ }^{*} R\right)$ which associates with each object $A \in V(R)$ its nonstandard extension ${ }^{*} A \in V\left({ }^{*} R\right)$. The nonstandard universe (whose members are known as the internal sets) is given by

$$
{ }^{*} V=\left\{x: x \in{ }^{*} A \text { for some } A \in V\right\}=\cup_{n=0}^{\infty}{ }^{*} V_{n}(R)
$$

The Transfer Principle states that any bounded quantifier statement holds in $V$ iff it holds in ${ }^{*} V$. (A bounded quantifier statement is a mathematical statement which can be written so that all quantifiers range over prescribed sets. This includes most statements used in practice.) This principle enables us to "switch" from the "standard world" $V$ to internal objects (elements of ${ }^{*} V$ ) in "the non-standard world" and back again: in proofs we can therefore "translate" a statement into the language of internal sets, manipulate it within ${ }^{*} V$ and then translate the results into the context of $V$. Note that not all sets in ${ }^{*} V(R)$ are internal. For example $R$ 
itself cannot be internal since it is a bounded subset of * $R$ yet it does not have any supremum. The sets that are not internal are called external. One has to be carefull working with external sets when the transfer principle is applied. For development of nonstandard probability theory we also need the $\omega_{1}$-saturation principle to hold. If $\left(A_{n}\right)_{n \in N}$ is a decreasing sequence of nonempty internal sets then their intersection is nonempty as well, $\cap_{n \in N} A_{n} \neq \emptyset$. Regarding infinitesimal analysis we will follow the basic notation used by Robinson (1961) but will try to recall the definitions and basic theorems whenever needed.

The advantage of NSA is that objects can be built using infinitesimally small or infinitely large numbers. The objects can then be lifted back to the "normal" mathematical world if needed. Many papers have been devoted to various lifting techniques. However this effort might be considered as anachronism from the perspective of applications or our understanding to the real world. If our objects constructed in ${ }^{*} V(R)$ provide a reasonable and applicable model of the investigated reality then why should we lift it back to the standard universe $V(R)$ ? Hence we will focus more on understanding and applicability of our constructions and will not care too much whether the results and objects can be translated back into the fully standard set-up.

\section{Infinitesimal Stochastic Calculus}

We will built stochastic processes on hyperfinite binomial trees. Let $T>0$ be a given terminal time and $H \in{ }^{*} N-N$ infinite. We set $\delta t=\frac{T}{H}$ to be our elementary infinitesimal time step and $\mathbf{T}=\{0, \delta t, 2 \cdot \delta t, \ldots, H \cdot \delta t=T\}$ to be our hyperfinite time line. We reserve $\delta t$ for that particular infinitesimal time step. On the other hand by $d t$ we will mean a general infinitesimal time step, possibly $\delta t$, but sometimes its infinite multiple, yet still infinitesimally small. While in NSA literature the stochastic processes are built on the space $\{-1,+1\}^{\mathbf{T}}$ of paths of lengths $T$ throughout the hyperfinite binomial tree we will rather use the set $\mathcal{T}=\{-1,+1\}^{\leq \mathbf{T}}=\cup_{t \in \mathbf{T}}\{-1,+1\}^{\mathbf{T} \cap(0, t]}$ corresponding to individual nodes of the tree. For $\omega \in \mathcal{T}$ we denote $t(\omega)$ to be the length of $\omega$, i.e. $t(\omega)=\max (\operatorname{dom}(\omega)) \in \mathbf{T}$ if $\omega$ is nonemepty, and 0 if $\omega$ is empty (i.e. if it is the root). The nodes with length $t(\omega)$ branch into two subsequent nodes $\omega \smile\{+1\}$ and $\omega \smile\{-1\}$. For a $t \in \mathbf{T}$ it will be useful to set $\mathcal{T}(t)=\{\omega \in \mathcal{T}: t(\omega)=t\}$ and $\mathcal{T}(<t)=\{\omega \in \mathcal{T}: t(\omega)<t\}$ If $\tau<t(\omega)$ then we denote $\omega \uparrow \tau=\omega \uparrow(\mathbf{T} \cap(0, \tau])$ to be the trucated path of length $\tau \in \mathbf{T}$.

In general we are going to study stochastic processes modelled by internal functions $X: \mathcal{T} \rightarrow{ }^{*} R$. For $\omega \in \mathcal{T}$ the value $X(\omega)$ naturally depends only on the information encoded in $\omega$ up to the time $t(\omega)$ and we do not have to bother with filtrations to take care of the issue of anticipation. Sometimes for $\tau \leq t(\omega)$ we will 
use the notation $X(\omega, \tau)$ instead of $X(\omega \uparrow \tau)$. If $\omega \in \mathcal{T}(T)$ then $\langle X(\omega, t)$ for $t \in \mathbf{T}\rangle$ is a realization of the stochastic process $x$ corresponding to the path $\omega$. On the other hand for a $t \in \mathbf{T}$ we denote $X(t)$ or $X_{t}$ to be the random variable $X(\omega, t)$ with $\omega \in \mathcal{T}(t)$.

Of course we are interested in processes that are generated by a law applied stepby-step throughout the binomial trees. Building a stochastic process we start with an initial value assigned to the root $X(\emptyset)=X_{0}$. For each already calculated $X(\omega)$ of length smaller than $T$ we define $X(\omega \frown\{+1\})=X_{u}(\omega)$ and $X(\omega \frown\{-1\})=X_{d}(\omega)$ on the subsequent nodes. Hence the two values +1 and -1 correspond to the coin tossing random element. The up and down values $X_{u}(\omega)$ and $X_{d}(\omega)$ are generally (internal) functions of the values of $X$ along $\omega$ or of values of other stochastic processes up to time $t=t(\omega)$, in most cases just of the values $X(\omega)$ and $t(\omega)$.

The most wellknown process is the Brownian motion first constructed using NSA infinitesimals by Anderson (1976). The original idea of Brown as well as the first applications of Bacheliur and Einstein were in fact based on the intuitive notion of infinitesimals. Set $Z(\emptyset)=0$ and $Z(\omega \frown\{j\})=Z(\omega)+j \cdot \sqrt{\delta t}$ where $j=+1,-1$. Consequently $Z(\omega)=\sum_{s \in \mathbf{T} \cap(0, t(\omega)]} \omega(s) \sqrt{\delta t}$. It will be useful to denote $\delta Z=j \sqrt{\delta t}$ for $j=+1,-1$. Then any stochastic process $X: \mathcal{T} \rightarrow{ }^{*} R$ can be in fact expressed by the equation $\delta X(\omega)=a(\omega) \delta t+b(\omega) \delta Z$ where $\delta X=X(\omega \frown\{j\}-X(\omega)$ describing how to generate its values from the initial one $X(\emptyset)=X_{0}$. The (internal) functions $a, b: \mathcal{T}(<T) \rightarrow{ }^{*} R$ can be obtained solving for every $\omega$ the two equations with two unknowns:

$$
\begin{gathered}
X(\omega \frown\{+1\})-X(\omega)=a(\omega) \delta t+b(\omega) \sqrt{\delta t}, \text { and } \\
X(\omega \frown\{-1\})-X(\omega)=a(\omega) \delta t-b(\omega) \sqrt{\delta t} .
\end{gathered}
$$

So far we did not need any probability measure, but to characterize distribution of of the process $Z$ or $X$ we need to introduce one. The advantage of the hyperfinite probability theory is that any probability measure $P$ on a hyperfinite space $\Omega$ is given by elementary probabilities on individual scenarious $\omega \in \Omega$. The probability measure is then in a straightforward manner defined on all internal subsets of $\Omega$ and can be extended using Caratheodory theorem and the $\omega_{1}$-saturation principle (see Loeb $(1975)$ ) to a standard measure denoted $L(P)$ which is $\sigma$-complete and takes standard values in $R_{0}^{+}$.

In the case of hyperfinite binomial trees in line with our intuition it is enough to specify the branching probabilities on $\mathcal{T}(<T)$. I.e. if an internal function specifies for every $\omega \in \mathcal{T}(<T)$ the probability $p_{+1}(\omega)$ of the process to go up, and if we set $p_{-1}(\omega)=1-p_{+1}(\omega)$ to be the complementary down/branching probability, then 
FiguRE 1. Binomial branching probabilities

we can define the elementary probability for every $\omega \in \mathcal{T}$ with $t=t(\omega)$ setting

$$
p(\omega)=\prod_{s \in \mathbf{T} \cap[0, t)} p_{\omega(s)}(\omega) .
$$

Equivalently the measure can be specified by an internal function $p: \mathcal{T}(T) \rightarrow$ ${ }^{*} R_{0}^{+}$such that $\sum_{\omega \in \mathcal{T}(T)} p(\omega)=1$. Then for $t<T, t \in \mathbf{T}$ we can extend the probability on any $\omega \in \mathcal{T}(t)$ setting

$$
p(\omega)=\sum_{\omega^{\prime} \in \mathcal{T}(T), \omega^{\prime} \mid t=\omega} p\left(\omega^{\prime}\right) .
$$

The corresponding branching probabilities can be defined by the equation $p_{+1}(\omega)=$ $\frac{p(\omega-\{+1\}}{p(\omega)}$ for $\omega \in \mathcal{T}(<T)$. Consequently any measure on $\mathcal{T}$ is given by a branching probability function. The basic measure on $\mathcal{T}$ is the uniform counting measure corresponding to constant branging probabilities equal to $\frac{1}{2}$. Then $p(\omega)=2^{-H}=$ $\frac{1}{|\mathcal{T}|}$ for every $\omega \in \mathcal{T}(T)$. It can be shown (Anderson (1975)) that with respect to this measure the process $Z$ has the properties of a Brownian motion, or more precisely that the lifted process $z\left(\omega,{ }^{o} t\right)={ }^{o} Z(\omega\lceil t), \omega \in \mathcal{T}(T)$ satisfies the classical conditions put on a Brownian motion. ${ }^{1}$

The key property we need to verify is that the increments $Z\left(\omega_{1}\right)-Z\left(\omega_{0}\right)$ are normally distributed, where $\omega_{0}$ fixed and $\omega_{1}$ such that $\omega_{1} \uparrow t\left(\omega_{0}\right)=\omega_{0}$. This easily follows from a hyperfinite version of the Central Limit Theorem (see Cutland (1991)) which will also serve as our key tool. The theorem is obtained simply applying the transfer principle to the classical Central Limit Theorem.

Theorem 3.1. Let $\left\{X_{n}: n \leq M\right\}$ with $M \in{ }^{*} N-N$ be an internal sequence of $*$-independent random variables on a hyperfinite probability space $\{\Omega, P\}$ with a common distribution function $F$ that has mean 0 and standard deviation 1 . Then the variable

$$
X=\frac{1}{\sqrt{M}} \sum_{n=1}^{M} X_{n}
$$

\footnotetext{
${ }^{1}$ We say that $x$ and $y$ in ${ }^{*} R$ are infinitesimally close $x \approx y$ if $x-y$ is infinitesimal. We say that $x \in{ }^{*} R$ is finite if $|x|<n$ for some $n \in N$. It can be easily shown that for every finite $x \in{ }^{*} R$ there is unique number $r \in R$ such that $x \approx r$. The number, which can be viewed as a result of infinitesimal rounding to the nearest standard number, is called the standard part of $x$ and denoted ${ }^{o} x=\operatorname{st}(x)$.

We say that a function $F: \mathbf{T} \rightarrow^{*} R$ is $\mathcal{S}$-continuous if $F(x) \approx F(y)$ whenever $x \approx y$. It can be shown that for almost all $\omega \in \mathcal{T}(T)$ with respect to the Loeb measure the functions $Z(\omega, \cdot)$ are $\mathcal{S}$-continuous. Hence for almost all $\omega$ the definition of $z(\omega, t)$ is correct.
} 
is normally distributed in the sense that $P[X \leq x] \approx{ }^{*} \psi(x)$ for any $x \in{ }^{*} R$ where

$$
\psi(x)=\frac{1}{\sqrt{2 \pi}} \int_{-\infty}^{x} e^{-\frac{x^{2}}{2}} d x
$$

is the cumultive standardized normal distribution function.

In the case of (Anderson's) Brownian motion the theorem can be directly applied to the independent incremental random variables $X_{n}=+1$ or -1 with probabilities $\frac{1}{2}$. It follows that the variable $z$ on $\mathcal{T}(T)$ which can be expressed as

$$
Z=\sum_{n=1}^{H} \sqrt{\delta t} X_{n}=T \cdot \frac{1}{\sqrt{H}} \sum_{k=1}^{H} X_{k}
$$

has the normal distribution (up to an infinitesimal error) with mean 0 and variance $T$. Moreover if for a given infinitesimal $d t$ being an infinite multiple of $\delta t$ and for a given $\omega \in \mathcal{T}(<T)$ we set $d z=d z\left(\omega^{\prime}\right)=Z\left(\omega \frown \omega^{\prime}\right)-Z(\omega)$ for $\omega^{\prime}$ of length $d t$ then $d z$ is also normally distributed with mean 0 and variance $d t$ but in this case up to an error of order $o(d t) .{ }^{2}$ Consequently we have a nutural interpretation of stochastic equations of the form

$$
d x=a \cdot d t+b \cdot d z
$$

with $a, b$ being finite constants or reasonable functions depending on $x$ and $t$ (Itô's processes). The process can be exactly defined on the elementary time step level by the equation $\delta x=a \cdot \delta t+b \cdot \delta z$ and at the same time we can say that $d x=a \cdot d t+b \cdot d z$ with an error of $o(d t)$. It is useful to prove the following lemma which says that when building step-by-step a stochastic process errors of the order $o(1) \delta t+O(\sqrt{\delta} \delta Z$ where $O(\sqrt{\delta}$ is a reasonable function do not matter.

Lemma 3.2. Let $X: \mathcal{T} \rightarrow{ }^{*} R$ be a stochastic process such that $X(\emptyset) \approx 0$ and $\delta X(\omega)=a(\omega) \delta t+b(\omega) \delta Z$ where $a(\omega) \approx 0$ for all $\omega$ and $b$ has the form $b=b(x, t)=$ $\beta(x, t) \sqrt{\delta t}$ with $\beta(x, t)$ Lipschitz continuous in $x$ and $t .{ }^{3}$ Then $X \approx 0$ for any a.s. on $\mathcal{T}$ with the uniform measure. Moreover if $b=b(\omega)=o(\sqrt{\delta t})$ then $X(\omega) \approx 0$ for all $\omega \in \mathcal{T}$.

Proof. (1) Let us assume that $X$ satisfies the equation $\delta X(\omega)=a(\omega) \delta t$ with $a(\omega) \approx$ 0 for all $\omega$. If $\epsilon=\max _{\omega \in \mathcal{T}}|a(\omega)|$ then $\epsilon \approx 0$ as the maximum is attained by an $\omega$ and so

$$
|X(\omega)| \leq|X(\emptyset)|+\sum_{s<t(\omega), s \in \mathbf{T}}|\delta(\omega \uparrow s)| \leq|X(\emptyset)|+\frac{t(\omega)}{\delta t} \epsilon \cdot \delta t \approx 0 .
$$

\footnotetext{
${ }^{2}$ We say that a number $x$ is of order $o(y)$ if $y \neq 0$ and $\frac{x}{y}$ is infinitesimal. We say that $x$ is of order $O(y)$ if $y \neq 0$ and $\frac{x}{y}$ is finite.

${ }^{3}$ I.e. there is a constant $c$ so that $\left|\beta\left(x_{2}, t_{2}\right)-\beta\left(x_{1}, t_{1}\right)\right| \leq c\left(\left|x_{2}-x_{1}\right|+\left|t_{2}-t_{1}\right|\right)$ for any $x_{1}, x_{2}, t_{1}, t_{2}$.
} 
(2) Let us assume that $X$ satisfies $\delta X=b \cdot \delta Z$ where $b \approx 0$ is a constant. Then $X(\omega)=X(\emptyset)+b \cdot Z(\omega)$ and so $X \approx 0$ a.s.

(3) Let us now assume that $\delta X=b \cdot \delta Z$ where $b(\omega) \approx o(\sqrt{\delta t})$. If $\epsilon=\max _{\omega \in \mathcal{T}}\left|\frac{b(\omega)}{\sqrt{\delta t}}\right|$ then $\epsilon \approx 0$ and $|X(t)| \leq|X(\emptyset)|+\epsilon \cdot \sqrt{\delta t} \cdot \sqrt{\delta t} \cdot \frac{t}{\delta t} \approx 0$.

(4) Let $\delta X(\omega)=\beta(t(\omega)) \sqrt{\delta t} \delta Z$ where $\beta(t)$ is a Lipschitz continuous function on $[0, T]$. Then for every $n \in N$ there is a finite division of the interval $[0, T]$ and a corresponding piecewise function $\beta_{n}(t)$ approximating $\beta(t)$ so that $\left|\beta_{n}(t)-\beta(t)\right| \leq \frac{1}{n}$ for every $t$. The process $X_{n}$ defined by setting $X_{n}(\emptyset)=X(\emptyset)$ and $\delta X_{n}=\beta_{n}(t) \sqrt{\delta t}$. $\delta Z$ can be expressed as a finite composition of Brownian motions with infinitesimal multipliers. Consequently according to $(2) X_{n} \approx 0$ a.s. Let $A_{n}=\{\omega \in \mathcal{T}$ : $\left.X_{n}(\omega) \not \approx 0\right\}$. Then $\cup_{n \in N} A_{n}$ has measure zero and for $\omega \in \mathcal{T}-\cup_{n \in N} A_{n}$ we have $\left|X_{n}(\omega)-X(\omega)\right| \leq \frac{t}{n}$ for every $n \in N$ and so $X(\omega) \approx 0$.

(5) Finally to prove the main statement of the Lemma decompose $X$ into its deterministic and stochastic part. All we need to prove is that the stochastic part is infinitesimally small a.s. So assume that $X(\emptyset)=0$ and $\delta X=\beta(X, t) \sqrt{\delta t} \cdot \delta Z$. Let $X_{0}$ be the process defined by $X_{0}(\emptyset)=0$ and $\delta X_{0}=\beta(0, t) \sqrt{\delta t} \cdot \delta Z$. According to (4) $X_{0} \approx 0$ a.s. Let us set $Y=X-X_{0}$ and prove that $Y \approx 0$. We know that $Y(\emptyset)=0, \delta Y=\left(\beta\left(X_{0}+Y, t\right)-\beta(0, t)\right) \sqrt{\delta t} \cdot \delta Z$, and so $|\delta Y| \leq c\left(\left|X_{0}\right|+|Y|\right) \delta t$ by the Lipschitz continuity of $\beta$. Let $\omega \in \mathcal{T}$ be such that $X_{0}(\omega, t) \approx 0$ for every $t \in \mathbf{T}$. Set $\epsilon=\max _{t \in \mathbf{T}}\left|X_{0}(\omega, t)\right| \approx 0$. Then we may estimate inductively $Y_{k}=Y(\omega \uparrow(k \cdot \delta t))$ inductively for $k=1, \ldots, H$ by

$$
\left|Y_{k}\right| \leq\left|Y_{k-1}\right|+\left(\epsilon+\left|Y_{k-1}\right|\right)(c \cdot \delta t)
$$

Consequently

$\left|Y_{k}\right| \leq c \cdot \epsilon \cdot \delta t \sum_{j=0}^{k-1}(k-j)(c \cdot \delta t)^{k} \leq c \cdot \epsilon \cdot k \cdot \delta t \sum_{j=0}^{k-1}(c \cdot \delta t)^{k}=c \cdot \epsilon \cdot t \frac{1-(c \cdot \delta t)^{k}}{1-(c \cdot \delta t)} \approx 0$

Corollary 3.3. If $X$ and $Y$ are two stochastic processes such that $X(\emptyset) \approx Y(\emptyset)$ and $D=X-Y$ satisfies $\delta D=o(1) \delta t+o(\sqrt{\delta t}) \delta Z$ then $X \approx Y$. If $\delta D=o(1) \delta t+$ $b(D, t) \sqrt{\delta t} \cdot \delta Z$ with $b$ Lipschitz continuous in the two variables then $X \approx Y$ a.s.

\section{Change of measure}

Let us firstly investigate what happens with the mean, variance, and the probability distribution of the Brownian motion $Z$ when the uniform branching probability $p_{+1}=\frac{1}{2}$ is changed to $\frac{1}{2}+\delta p$ for a $\delta p \approx 0$. The expected value and variance of $\delta Z$ under the new measure $Q$ is

$$
E_{Q}[\delta Z]=\left(\frac{1}{2}+\delta p\right) \cdot \sqrt{\delta t}+\left(\frac{1}{2}-\delta p\right) \cdot(-\sqrt{\delta t})=2 \delta p \sqrt{\delta t}
$$


$\operatorname{Var}_{Q}[\delta Z]=E_{Q}\left[\delta Z^{2}\right]-E_{Q}[\delta Z]^{2}=\left(\frac{1}{2}+\delta p\right) \cdot \delta t+\left(\frac{1}{2}-\delta p\right) \cdot \delta t-4 \delta p^{2} \cdot \delta t=\delta t\left(1-4 \delta p^{2}\right)$. If $\delta p$ is constant then the random variable $Z(T)-Z(0)$ can be expressed as $\sum_{n=0}^{H-1} \delta Z_{n}$ where $\delta Z_{n}$ are independent and all have the same distribution with one value $+\sqrt{\delta t}$ attained with probability $\frac{1}{2}+\delta p$ and the other value $-\sqrt{\delta t}$ attained with probability $\frac{1}{2}-\delta p$. Consequently

$$
E_{Q}[Z(T)-Z(0)]=H \cdot 2 \cdot \delta p \sqrt{\delta t}=T \cdot \frac{2 \delta p}{\sqrt{\delta t}}
$$

and

$$
\operatorname{Var}_{Q}[Z(T)-Z(0)]=T\left(1-4 \delta p^{2}\right) .
$$

Since we restrict ourselves only to processes with finite mean we need $\frac{2 \delta p}{\sqrt{\delta t}}$ to be finite, i.e. $\delta p=\frac{\alpha}{2} \sqrt{\delta t}$ for a finite $\alpha$. In other words only changes of order $\sqrt{\delta t}$ are admissible. Notice that in this case the mean of the process $E_{Q}[Z(T)-Z(0)]=\alpha T$ can be changed to an arbitrary finite number, but the variance $\operatorname{Var}_{Q}[Z(T)-Z(0)]=$ $T\left(1-\alpha^{2} \delta t\right) \approx T$ remains unchanged up to an infinitesimal error.

Moreover the distribution of $Z(T)$ remains normal. Let

$$
X_{n}=\frac{\delta Z_{n}-\alpha \delta t}{\sqrt{\delta t}} .
$$

Then $X_{n}$ are independent with the same distribution with mean 0 and variance 1 . Consequently we may apply the hyperfinite central limit theorem to

$$
\frac{1}{\sqrt{H}} \sum_{n=1}^{H} X_{n}=\frac{Z(T)-Z(0)-\alpha T}{\sqrt{T}} .
$$

So we prove that $Z(T)-Z(0)$ is a normally distributed variable (up to an infinitesimal error) with mean $\alpha T$ and variance $T$. The same analysis applies to the differential $d Z$ for an infinitesimal $d t$ that is at the same time an infinite multiple of $\delta z$. Thus the stochastic process $z$ with respect to the changed measure satisfies the classical stochastic differential equation $d x=\alpha \cdot d t+d z$ with $d z$ normally distributed with mean 0 and variance $d t$. Note that we have got at least two different representations of a (real world) process satisfying this equation: first the process with drift $\alpha$ defined on $\mathcal{T}$ with the uniform measure and then the original Brownian motion on $\mathcal{T}$ with the changed measure.

On the other hand if there is a process $X$ with a drift $\beta$ on $\mathcal{T}$ with the uniform measure, i.e. $\delta X=\beta \cdot \delta t+\delta Z$ then we may change the measure (as above with the coefficient $\alpha=-\beta$ ) so that $X$ is a martingale (has zero drift) with respect to the changed measure. To show that this a special case of an equivalent martingale 
measure we yet need to show that the changed measure is equivalent to the uniform measure on $\mathcal{T} .4$

The density of the changed measure $Q$ with respect to the uniform measure $P$ can be for an $\omega \in \mathcal{T}(t)$ expressed as

$$
\frac{Q(\omega)}{P(\omega)}=\prod_{s \in \operatorname{dom}(\omega)}(1+\alpha \omega(s) \sqrt{\delta t}) .
$$

To understand to the expression on the right hand side we need the following (see also Cutland at al. (1991))

Lemma 4.1. For any $\omega \in \mathcal{T}(t)$ such that $|Z(\omega)|$ is finite and for any finite constants $\alpha$ and $\beta$

$$
\prod_{s \in \operatorname{dom}(\omega)}(1+\alpha \cdot \omega(s) \sqrt{\delta t}+\beta \cdot \delta t) \approx \exp \left(\alpha Z(\omega)+\left(\beta-\frac{\alpha^{2}}{2}\right) t\right) .
$$

Proof. If we apply the logarithm to the left hand side of the equation then

$$
\begin{array}{r}
\left.\ln \left(\prod_{s \in \operatorname{dom}(\omega)}(1+\alpha \cdot \omega(s) \sqrt{\delta t}+\beta \cdot \delta t)\right)\right)= \\
\left.\sum_{s \in \operatorname{dom}(\omega)} \ln (1+\alpha \cdot \omega(s) \sqrt{\delta t}+\beta \cdot \delta t)\right) .
\end{array}
$$

The Taylor expansion of $\ln (1+x)$ is $\ln (1+x)=x-\frac{x^{2}}{2}+O\left(x^{3}\right)$, hence

$$
\begin{array}{r}
\ln (1+\alpha \cdot \omega(s) \sqrt{\delta t}+\beta \cdot \delta t)= \\
\alpha \cdot \omega(s) \sqrt{\delta t}+\beta \cdot \delta t-\frac{1}{2}\left(\alpha^{2} \delta t+2 \alpha \beta \omega(s) \delta t^{3 / 2}+\beta^{2} \delta t^{2}\right)+O\left(\delta t^{3 / 2}\right)= \\
\alpha \cdot \omega(s) \sqrt{\delta t}+\left(\beta-\frac{1}{2} \alpha^{2}\right) \delta t+o(\delta t) .
\end{array}
$$

Since the $o(\delta t)$ terms can be in the sum over $\operatorname{dom}(\omega)$ neglected we get

$$
\left.\sum_{s \in \operatorname{dom}(\omega)} \ln (1+\alpha \cdot \omega(s) \sqrt{\delta t}+\beta \cdot \delta t)\right) \approx \alpha \cdot Z(\omega)+\left(\beta-\frac{1}{2} \alpha^{2}\right) t .
$$

Since exp is $\mathcal{S}$-continuous we have proved (4.1).

Theorem 4.2. The changed measure $Q$ is equivalent to the uniform measure $P$ on $\Omega=\mathcal{T}(T)$.

Proof. Let us assume $A \subseteq \Omega$ is such that $P(A) \gtrsim 0$. Since $Z(\omega)$ is finite $L(P)$ almost surely there is an $n \in N$ such that $A_{n}=\{\omega \in A:|Z(\omega)|<n\}$ is also positive, i.e. $P\left(A_{n}\right) \gtrsim 0$. It follows from the lemma that there is a finite constant $K$ such that $\exp (-K) \leq \frac{Q(\omega)}{P(\omega)} \leq \exp (K)$ for every $\omega \in A_{n}$. Consequently $Q(A) \geq$

${ }^{4}$ Generally we say that two measures $P$ and $Q$ on a hyperfinite space $\Omega$ are equivalent if $P(A) \approx 0$ whenever $Q(A) \approx 0$ for any internal $A \subseteq \Omega$. 
$Q\left(A_{n}\right) \geq \exp (-K) \cdot P\left(A_{n}\right) \gtrsim 0$. Similarly we can prove that if $Q(A) \gtrsim 0$ then $P(A) \gtrsim 0$ as well.

Generally we wish to consider change of measure determined by a non constant adjustments on the binomial branches, i.e. the change of $p_{+1}=\frac{1}{2}$ to $\frac{1}{2}(1+\alpha(\omega) \sqrt{\delta t})$ where $\alpha: \mathcal{T}(<T) \rightarrow{ }^{*} R$ is an internal function taking only (or at least $L(P)$ surely) finite values. In the sense of the analysis above the "Brownian" motion with respect to this measure is equivalent to the process defined by $\delta X(\omega)=\alpha(\omega) \cdot \delta t+\delta Z$ on $\mathcal{T}$ with the uniform measure. If $\alpha$ is a continuous deterministic function of $t=t(\omega)$ then the analysis above can be easily generalized: $X(t)-X(0)$ is normal (up to an infinitesimal error) with variance $t$ and mean equal to

$$
\int_{0}^{t} \alpha(s) d s \approx \sum_{s \in \mathbf{T} \cap[0, t)} \alpha(s) \delta t .
$$

The changed measure $Q$ is in this case again equivalent to the uniform measure $P$. Nevertheless if $\alpha$ depends on $\omega \in \mathcal{T}(t)$ we cannot generally conclude that the process $\delta X(\omega)=\alpha(\omega) \cdot \delta t+\delta Z$ leads to a normal ditribution. Note that the process is nevertheless well defined for any internal function $\alpha$. We will however restrict our attention only to those drift functions $\alpha$ resulting in realistic stochastic processes that are almost surely finite and continuous.

Let us consider the geometric Brownian motion frequently used for stock price modelling. It is given by the equation $d S=\mu \cdot S \cdot d t+\sigma \cdot S \cdot d z$ where $\mu$ is the relative drift and $\sigma$ the volatility. The process is constructed inductively on $\mathcal{T}$ with the uniform measure setting $S(\emptyset)=S_{0}$ and $S(\omega \frown\{j\})=S(\omega)+\mu S(\omega) \delta t+\sigma S(\omega) j \sqrt{\delta t}$. Note that in this case the coefficients of $\delta t$ and $\delta Z$ are stochastic (depend on $\omega \in$ $\mathcal{T}(t))$. The resulting distribution indeed is not normal, but lognormal. To show that $S(T)$ is lognormal set $X=\ln S$, then using the technique of the proof of Lemma 4.1 we get that

$$
X(\omega \smile\{j\})-X(\omega)=\left(\mu-\frac{\sigma^{2}}{2}\right) \delta t+\sigma j \sqrt{\delta t}+o(\delta t) .
$$

According to Lemma 3.2 the process $X$ has (up to an infinitesimal) the same distribution as the process given by $\delta X^{\prime}=\left(\mu-\frac{\sigma^{2}}{2}\right) \delta t+\sigma \delta Z$. Consequently $X(T)-$ $X(0)=\ln \frac{S(T)}{S_{0}}$ has a normal distribution with mean $\left(\mu-\frac{\sigma^{2}}{2}\right) T$ and standard deviation $\sigma \sqrt{T}$. In other words $S(T)$ is (up to an infinitesimal error) lognormally distributed.

Equivalently we may set the values of the process without any drift, $S(\emptyset)=S_{0}$ and $S(\omega-\{j\})=S(\omega)+\sigma S(\omega) j \sqrt{\delta t}$, and change the measure on $\mathcal{T}$ to achieve the desired drift of $S$. To set up the adjustment $\alpha \frac{\sqrt{\delta t}}{2}$ on the node $\omega$ we need the 
following equation to hold

$$
\mu S \delta t=E_{Q}(\delta S)=\left(\frac{1}{2}+\alpha \frac{\sqrt{\delta t}}{2}\right) \sigma S \sqrt{\delta t}-\left(\frac{1}{2}-\alpha \frac{\sqrt{\delta t}}{2}\right) \sigma S \sqrt{\delta t}=\alpha \sigma S \delta t .
$$

Consequently if we set $\alpha=\frac{\mu}{\sigma}$ and change the uniform branching probabilities from $\frac{1}{2}$ to $\frac{1}{2}+\alpha \frac{\sqrt{\delta t}}{2}$ then the process $S$ with respect to the measure also satisfies the stochastic differential equation $d S=\mu \cdot S \cdot d t+\sigma \cdot S \cdot d z$. Note that the measure is again equivalent since $\alpha=\frac{\mu}{\sigma}$ is finite and constant.

\section{Equivalent Martingale Measure}

Let us consider a general derived security $f$ depending on $n$ underlying assets corresponding to $n$ primary sources of uncertainty $z_{1}, \ldots, z_{n}$ modelled as independent Brownian motions. Let us assume that $f$ satisfies the stochastic differential equation of the form

$$
d f=\mu \cdot f \cdot d t+\sigma_{1} \cdot f \cdot d z_{1}+\cdots+\sigma_{n} \cdot f \cdot d z_{n} .
$$

To model such a process let $\mathcal{T}_{1}, \ldots, \mathcal{T}_{n}$ be $n$ copies of the hyperfinite binomial tree on $\mathbf{T}$. Let

$$
\mathcal{T}_{1} \otimes \cdots \otimes \mathcal{T}_{n}=\left\{\left\langle\omega_{1}, \ldots, \omega_{n}\right\rangle: \omega_{1} \in \mathcal{T}_{1}, \ldots, \omega_{n} \in \mathcal{T}_{n}, \text { and } t\left(\omega_{1}\right)=\cdots=t\left(\omega_{n}\right)\right\} .
$$

According to (5.1) we build the process as follows: $F(\emptyset)=F_{0}$ and

$$
\begin{gathered}
F\left(\left\langle\omega_{1}^{\Upsilon}\left\{j_{1}\right\}, \ldots, \omega_{n}^{\Upsilon}\left\{j_{n}\right\}\right\rangle\right)= \\
F\left(\left\langle\omega_{1}, \ldots, \omega_{n}\right\rangle\right) \cdot\left(1+\mu \delta t+\sigma_{1} j_{1} \sqrt{\delta t}+\cdots+\sigma_{n} j_{n} \sqrt{\delta t}\right) .
\end{gathered}
$$

Given measures $P_{1}, \ldots, P_{n}$ on $\mathcal{T}_{1}, \ldots, \mathcal{T}_{n}$ we define the product measure $P=P_{1} \otimes$ $\cdots \otimes P_{n}$ by $P\left(\left\langle\omega_{1}, \ldots, \omega_{n}\right\rangle\right)=P_{1}\left(\omega_{1}\right) \cdots P_{n}\left(\omega_{n}\right)$. We again start with the uniform product measure corresponding to $\frac{1}{2}$ branching probabilities throughout each of the hyperfinite binomial trees. Then we are going to change the up-branching probabilities along $\mathcal{T}_{i}$ to $\frac{1}{2}+\alpha_{i} \frac{\sqrt{\delta t}}{2}$ for some finite $\alpha_{i}$. In other words we change $P_{i}$ to $Q_{i}$ and set $Q=Q_{1} \otimes \cdots \otimes Q_{n}$. If $X$ is the corresponding log-process, i.e. $\delta X=\mu \delta t+\sum \sigma_{i} \delta Z_{i}$, then $\left.E_{Q_{i}}[\delta X]=\left(\mu+\alpha_{i} \sigma_{i}\right) \sqrt{\delta t}, \operatorname{Var}_{[} Q_{i}\right](\delta X)=\sigma_{i}^{2} \delta t+o(\delta t)$ and since $\delta Z_{i}$ are independent

$$
\begin{gathered}
E_{Q}[\delta X]=\left(\mu+\alpha_{1} \sigma_{1}+\cdots+\alpha_{n} \sigma_{n}\right) \delta t, \\
\operatorname{Var}_{Q}[\delta X]=\left(\sigma_{1}^{2}+\cdots+\sigma_{n}^{2}\right) \delta t+o(\delta t) .
\end{gathered}
$$

Hence $F \approx \exp (X)$ satisfies the stochastic differential equation

$$
\frac{d f}{f}=\left(\mu+\alpha_{1} \sigma_{1}+\cdots+\alpha_{n} \sigma_{n}\right) d t+\sigma_{1} d z_{1}+\cdots+\sigma_{n} d z_{n} .
$$


We are almost ready to build up a general equivalent martingale measure. Let $g$ be a numeraire, i.e. a (almost surely) positive stochastic process with the $n$ sources of uncertainty satisfying the equation:

$$
\frac{d g}{g}=\mu_{g} d t+\sigma_{1, g} d z_{1}+\cdots+\sigma_{n, g} d z_{n}
$$

Let us start with its canonical representation on $\mathcal{T}_{1} \otimes \cdots \otimes \mathcal{T}_{n}$ with the uniform counting measure given by: $G(\emptyset)=G_{0}$ and

$$
\begin{gathered}
G\left(\left\langle\omega_{1}^{-}\left\{j_{1}\right\}, \ldots, \omega_{n}^{\Upsilon}\left\{j_{n}\right\}\right\rangle\right)= \\
G\left(\left\langle\omega_{1}, \ldots, \omega_{n}\right\rangle\right) \cdot\left(1+\mu_{g} \delta t+\sigma_{1, g} j_{1} \sqrt{\delta t}+\cdots+\sigma_{n, g} j_{n} \sqrt{\delta t}\right) .
\end{gathered}
$$

We would like to change the uniform measure to a measure $Q$ so that for any other derivative security $f$ with the same sources of uncertainty the ratio $\frac{f}{g}$ is a martingale, i.e. if $F$ is the canonical representation of $f$ then

$$
\frac{F_{0}}{G_{0}} \approx E_{Q}\left[\frac{F(t)}{G(t)}\right]
$$

for any $t \in \mathbf{T}$. At this point we need to introduce the notion of risk-free interest rate and the assumption of arbitrage-free markets. A derivative security or a portfolio of securitites is risk-less if its value is just a function of time. The arbitrage-free market assumption says that any risk-free portfolio earns just a risk-free return (otherwise there would be an arbitrage when a risk-free portfolio financed for a risk-free interest rate would earn a positive profit). Note that the (instantenous) risk-free interest rate itself is in general stochastic.

The arbitrage-free principle is used to prove that for each source of uncertainty $d z_{i}$ there is a price of risk $\lambda_{i}$ increasing the expected return of a security with $d z_{i}$-volatility $\sigma_{i}$ with respect to the risk-free interest rate by $\lambda_{i} \sigma_{i}$. I.e. if

$$
\frac{d f}{f}=\mu_{f} d t+\sigma_{1, f} d z_{1}+\cdots+\sigma_{n, f} d z_{n}
$$

then $\mu=r+\lambda_{1} \sigma_{1}+\cdots+\lambda_{n} \sigma_{n}$ where $r$ is the risk-free interest rate.

Let us assume without loss of generality that there are $n$ underlying securities $\theta_{1}, \ldots, \theta_{n}$ representing the $n$ sources of uncertainty. Let $\delta \theta_{i}=\mu_{i} \theta_{i} \delta t+\sigma_{i} \theta_{i} \delta Z_{i}$ with $\sigma_{i} \neq 0$ be the canonical representation of the $i$-th process on $\mathcal{T}_{i}$ for $i=1, \ldots, n$. Let us set $\lambda_{i}=\frac{\mu_{i}-r}{\sigma_{i}}$ and show that the prices of risk are also consistent with the return of $f$ canonically represented by $F$ on $\mathcal{T}_{1} \otimes \cdots \otimes \mathcal{T}_{n}$. Let $\Pi=f-\sum_{i=1}^{n} k_{i} \theta_{i}$ where $k_{i}=\frac{\sigma_{i, f} F(\omega)}{\sigma_{i} \theta_{i}(\omega)}$ be a portfolio (set at time $t=t(\omega)$ at the state $\omega$ ) consisting of a linear combination of the security $f$ and the underlying securities $\theta_{i}$ so that all the $d Z_{i}$ 's are eliminated - i.e. its values at all states at $t+\delta t$ are the same. Consequently the portfolio is risk-free and according to the arbitrage-free markets 
principle $\delta \Pi=r \Pi \delta t+o(\delta)$. Expanding the left hand side and the right hand side we get the equation

$$
\left(\mu_{f}-\sum_{i=0}^{n} \mu_{i} \frac{\sigma_{i, f}}{\sigma_{i}}\right) F \delta t=\left(r-\sum_{i=0}^{n} r \frac{\sigma_{i, f}}{\sigma_{i}}\right) F \delta t+o(\delta t),
$$

so

$$
\mu_{f}-r \approx \sum_{i=0}^{n}\left(\mu_{i}-r\right) \frac{\sigma_{i, f}}{\sigma_{i}}=\sum_{i=0}^{n} \lambda_{i} \sigma_{i, f}
$$

Note that when we change the uniform measure on $\mathcal{T}_{i}$ by adjusting the branching probability from $\frac{1}{2}$ to $\frac{1}{2}+\alpha_{i} \frac{\sqrt{\delta t}}{2}$ we are in fact changing the price of risk from the original $\lambda_{i}$ to $\lambda_{i}+\alpha_{i}$.

Theorem 5.1 (Equivalent Martingale Measure). Let $g$ be a numeraire represented by (5.3) on $\mathcal{T}_{1} \otimes \cdots \otimes \mathcal{T}_{n}$ with the uniform measure. Let $Q$ be the changed measure on $\mathcal{T}_{1} \otimes \cdots \otimes \mathcal{T}_{n}$ obtained by changing the splitting probilities on $\mathcal{T}_{i}$ from $\frac{1}{2}$ to $\frac{1}{2}+\alpha_{i} \frac{\sqrt{\delta t}}{2}$ with $\alpha_{i}=\sigma_{g, i}-\lambda_{i}$, i.e. changing the price of risk to $\sigma_{g, i}$. Then if $f$ is any stochastic process with the same sources of uncertainty and represented by the equation (5.2) then $\frac{f}{g}$ is a martingale with respect to $Q$, i.e. $\frac{F_{0}}{G_{0}} \approx E_{Q}\left[\frac{F(t)}{G(t)}\right]$ for all $t$.

We could show that $\frac{F}{G}$ is a martingale with respect to $Q$ just applying an elementary but tedious artithmetics of infinitesimals. It is however more elegant and also useful to prove a nonstandard version of the Itô's lemma first.

Lemma 5.2 (Itô). Let $G$ be a real function of two variables with partial derivatives of all orders. Let $X$ be a stochastic process on $\mathcal{T}_{1} \otimes \cdots \otimes \mathcal{T}_{n}$ following the equation $d x=a(x, t) d t+\sum_{i=1}^{n} b_{i}(x, t) d z_{i}$ where $b_{i}$ are Lipschitz continuous. Then the stochastic process $G=G(X, t)$ follows the equation

$$
d G=\left(\frac{\partial G}{\partial x} a+\frac{\partial G}{\partial t}+\frac{1}{2} \frac{\partial^{2} G}{\partial x^{2}} \sum_{n=1}^{n} b_{i}^{2}\right) d t+\frac{\partial G}{\partial x} \sum_{i=1}^{n} b_{i} d z_{i}
$$

Proof. Let us apply Taylor's expansion to $\delta G=G(x+\delta x, t+\delta t)-G(x, t)$ :

$$
\delta G=\frac{\partial G}{\partial x} \delta x+\frac{\partial G}{\partial t} \delta t+\frac{1}{2} \frac{\partial^{2} G}{\partial x^{2}} \delta x^{2}+o(|\delta x|+|\delta t|) .
$$

Let us plugin the equation $d X=a(X, t) \delta t+\sum_{i=1}^{n} b_{i}(X, t) \delta Z_{i}$ defining the process $X$ into the Taylor expansion. We use the facts that $\delta Z_{i}^{2}=\delta_{i}$ and $\delta Z_{i} \delta Z_{j}$ for $i \neq j$ takes only the two values $\delta t$ and $-\delta t$ with equal probabilities, hence has the mean 0 and variance $\delta t^{2}$. According to the Lemma 3.2 we may neglect deterministic elements of order less than $\delta t$ and under certain conditions stochastic elements with standard deviation of order $\delta t$. So

$$
\delta G=a \frac{\partial G}{\partial x} \delta t+\sum_{i=1}^{n} \frac{\partial G}{\partial x} b_{i}(X, t) \delta Z_{i}+\frac{\partial G}{\partial t} \delta t+
$$


CONSTRUCTION OF EQUIVALENT MARTINGALE MEASURES WITH INFINITESIMALS 15

$$
\begin{gathered}
\frac{1}{2} \frac{\partial^{2} G}{\partial x^{2}}\left(a^{2} \delta t+\sum_{i=1}^{n} b_{i}^{2} \delta t+\sum_{i \neq j} b_{i} b_{j} \delta Z_{i} \delta Z_{j}\right)+o(1) \delta t+O(1) \delta t \delta Z= \\
\left(a \frac{\partial G}{\partial x}+\frac{\partial G}{\partial t}+\frac{1}{2} \frac{\partial^{2} G}{\partial x^{2}} \sum_{i=1}^{n} b_{i}^{2}\right) \delta t+\sum_{i=1}^{n} \frac{\partial G}{\partial x} b_{i} \delta Z_{i}+o(1) \delta t+o(1) \delta Z
\end{gathered}
$$

Consequently in the sense of Lemma $3.2 G$ follows the equation (5.2).

To finalize our proof of the Equivalent Martingale Measure Theorem, i.e. to prove that $\frac{f}{g}$ is a martingale with respect to $Q$, we will use the the function the logarithmic transformation. First since $\sigma_{g, i}$ is w.r.t $Q$ the price of the i-th component of risk, the processes $F$ and $G$ satisfy the stochastic differential equations

$$
\begin{gathered}
d f=\left(r+\sum_{i=1}^{n} \sigma_{g, i} \cdot \sigma_{f, i}\right) f \cdot d t+\sum_{i=1}^{n} \sigma_{f, i} \cdot f \cdot d z_{i} \text { and } \\
d g=\left(r+\sum_{i=1}^{n} \sigma_{g, i}^{2}\right) g \cdot d t+\sum_{i=1}^{n} \sigma_{g, i} \cdot g \cdot d z_{i} .
\end{gathered}
$$

According to the Itô's lemma the processes $\ln F$ and $\ln G$ satisfy

$$
\begin{gathered}
d(\ln f)=\left(r+\sum_{i=1}^{n} \sigma_{g, i} \cdot \sigma_{f, i}-\frac{1}{2} \sum_{i=1}^{n} \sigma_{f, i}^{2}\right) d t+\sum_{i=1}^{n} \sigma_{f, i} \cdot d z_{i} \text { and } \\
d(\ln g)=\left(r+\frac{1}{2} \sum_{i=1}^{n} \sigma_{g, i}^{2}\right) d t+\sum_{i=1}^{n} \sigma_{g, i} \cdot d z_{i} .
\end{gathered}
$$

Hence $\ln \frac{f}{g}=\ln f-\ln g$ follows

$$
\begin{aligned}
d\left(\ln \frac{f}{g}\right)=(- & \left.\frac{1}{2} \sum_{i=1}^{n}\left(\sigma_{f, i}^{2}-2 \sigma_{g, i} \cdot \sigma_{f, i}+\sigma_{g, i}^{2}\right)\right) d t+\sum_{i=1}^{n}\left(\sigma_{f, i}-\sigma_{g, i}\right) d z_{i}= \\
& -\frac{1}{2} \sum_{i=1}^{n}\left(\sigma_{f, i}-\sigma_{g, i}\right)^{2} d t+\sum_{i=1}^{n}\left(\sigma_{f, i}-\sigma_{g, i}\right) d z_{i} .
\end{aligned}
$$

Finally let us apply the exponential function to $\ln \frac{f}{g}$ and the Itô's lemma again to get

$$
d\left(\exp \ln \frac{f}{g}\right)=d\left(\frac{f}{g}\right)=\sum_{i=1}^{n}\left(\sigma_{f, i}-\sigma_{g, i}\right) \frac{f}{g} d z_{i} .
$$

In terms of the representations $F$ and $G$ on $\mathcal{T}_{1} \otimes \cdots \otimes \mathcal{T}_{n}$ the equations hold up to an error of order $o(1) \delta+o(\sqrt{\delta t}) \delta Z$. Hence according to Lemma 3.2 we have proved that $\frac{F}{G}$ is a martingale, i.e. for every $t$ :

$$
\frac{F_{0}}{G_{0}} \approx E_{Q}\left(\frac{F(t)}{G(t)}\right)
$$




\section{REFERENCES}

1. Anderson, R.M. (1976), A Nonstandard Representation for Brownian Motion and Itô Integration, Israel Math.J., 25, 15-46.

2. Cox, J., S. Ross, and M. Rubisntein (1979), Option Pricing: A Simplified Approach,J. Financial Econom., 7, 229-263.

3. Cutland, N., E.Kopp, and W.Willinger (1991), A Nonstandard Approach to Option Pricing, Mathematical Finance, 1(4), 1-38.

4. Cutland, N., E.Kopp, and W.Willinger (1993), A nonstandard treatment of options driven by Poisson processes, Stochastics and Stochastics Reports, 42, 115-133.

5. Cutland, N., E.Kopp, and W.Willinger (1995), From Discrete to Continuous Stochastic Calculus,Stochastics and Stochastic Reports, 52, 173-192.

6. Cutland, N., E.Kopp, W.Willinger, and M.C.Wyman (1997), Convergence of Snell Envelopess and Critical Prices in the American Put, in Mathematics of Derivative Securitities (Eds. M.A.H. Dempster and S.R.Pliska), CUP, 126-140.

7. Cutland, N. (2000), Loeb Measures in Practice: Recent Advances, Lecture Notes in Mathematics, Springer.

8. Hull, J. (2006), Options, Futures, and Other Derivatives, Prentice Hall.

9. Hurd, A.E. and P.A. Loeb (1985), An Introduction to Nonstandard Real Analysis, Academic Press.

10. Keisler, J.H. (1984), An Infinitesimal Approach to Stochastic Analysis, Mem. Amer. Math. Soc., 297.

11. Keisler, J.H. (2000), Elementary Calculus - an Infinitesimal Approach, 2nd Edition, University of Wisconsin - Creative Commons.

12. Loeb, P.A. (1975), Conversion from Nonstandard to Standard Measure Spaces and Applications in Probability Theory, Trans. Amer. Math. Soc., 211, 113-122.

13. Robinson, A. (1961), Nonstandard Analysis, Proc. Roy. Acad. Amsterdam Ser. A, 64, 432440.

Faculty of Finance and Accounting, University of Economics, Winston Churchill Sq. 4, Prague 3, Czech Republic

E-mail address: witzanyj@vse.cz 